\title{
THE CONTINUOUS JACOBI TRANSFORM
}

\author{
E.Y. DEEBA \\ Department of Mathematical Sciences \\ University of Petroleum and Minerals \\ Dhahran, Saudi Arabia \\ and \\ E.L. KOH \\ Department of Mathematics and Statistics \\ University of Regina \\ Regina, Canada S4S 4J5 \\ (Received April 20, 1982 and in revised form October 15, 1982)
}

ABSTRACT. The purpose of this paper is to define the continuous Jacobi transform as an extension of the discrete Jacobi transform. The basic properties including the inversion theorem for the continuous Jacobi transform are studied. We also derive an inversion formula for the transform which maps $L^{1}\left(R^{+}\right)$into $L_{w}^{2}(-1,1)$, where $w(x)=(1-x)^{\alpha}(1+x)^{\beta}$.

KEY WORDS AND PHRASES. Continuous Jacobi transform, discrete Jacobi transform, continuous Legendre transform, inverse Jacobi transform.

1980 MATHEMATICS SUBJECT CLASSIFICATION CODES. 44A15, 44A20, 33A30, 33A65

\section{INTRODUCTION.}

This paper is directed to specialists in the theory and applications of integral transforms. However, familiarity with reference [1,2] would be useful for non-specialists interested in the paper.

Debnath [3-4] first studied the theory of the discrete Jacobi transform with applications to physical problems described by differential equations including the problem of heat conduction in a finite domain with variable thermal conductivity.

The purpose of this paper is to define the continuous Jacobi transform, study its basic properties and develop an inversion theorem. The continuous Jacobi 
transform generalizes, on the one hand, the continuous Legendre transform studied by Butzer, Stens and Wehrens [5] and on the other, the discrete Jacobi transform studied by Debnath [3]. The study of such transforms is interesting in its own right as well as in their applications to boundary value problems and in sampling theory.

The paper is divided as follows. Section two includes some facts of the hypergeometric function and basic relations that hold for the Jacobi transform of the first kind. Section three is devoted to the definition of the continuous Jacobi transform and the study of its basic properties. Moreover, in the same section, we derive an inversion formula for the transform.

\section{PRELIMINARIES}

In this section we discuss the basic background material necessary for the development of the continuous Jacobi transform.

Let $a, b, c$ be real numbers such that $c \neq 0,-1,-2, \ldots$. Then the hypergeometric function

$$
F(a, b ; c ; z)=\sum_{k=0}^{\infty} \frac{(a)_{k}(b) k}{(c)_{k} k !} z^{k},|z|<1,
$$

is absolutely and uniformly convergent on each compact subinterval of $(-1,1)$. Moreover, the series converges at $z=-1$ and $z=1$ provided that $c-a-b+1>0$ and $c-a-b>0$ respectively. In particular,

$$
\lim _{z \rightarrow 1^{-}} F(a, b ; c ; z)=F(a, b ; c ; 1)=\frac{\Gamma(c) \Gamma(c-a-b)}{\Gamma(c-a) \Gamma(c-b)} .
$$

We remark that the gamma function, whenever used, is a well-defined function of its argument.

The hypergeometric function (2.1) satisfies the following contiguous relations (see [6], [7]) which will be used throughout the sequel and are stated for the sake of completeness.

$$
\begin{aligned}
& F(a, b ; c ; z)=(1-z){ }^{c-a-b} F(c-a, c-b ; c ; z) ; \\
& F(a, b ; c ; z)=(1-z)^{-a} F\left(a, c-b ; c ; \frac{-z}{1-z}\right) ;
\end{aligned}
$$




$$
\begin{aligned}
& a F(a+1, b ; c ; z)=b F(a, b+1 ; c ; z)-(b-a) F(a, b ; c ; z) ; \\
& (c-b) z F(a, b ; c+1 ; z)=c F(a-1, b ; c ; z)-c(1-z) F(a, b ; c ; z) ; \\
& (c-a-b) F(a, b ; c ; z)=(c-b) F(a, b-1 ; c ; z)-a(1-z) F(a+1, b ; c ; z) ; \\
& (c-a-b) F(a, b ; c ; z)=(c-a) F(a-1, b ; c ; z)-b(1-z) F(a, b+1 ; c ; z) ; \\
& \frac{d^{n}}{d z^{n}} F(a, b ; c ; z)=\frac{(a)_{n}(b)}{(c)} F(a+n, b+n ; c+n ; z) .
\end{aligned}
$$

The Jacobi function, $\mathrm{P}_{\lambda}^{(\alpha, \beta)}(\mathrm{x})$, of the first kind is defined by

$$
\mathrm{P}_{\lambda}^{(\alpha, \beta)}(\mathrm{x})=\frac{\Gamma(\lambda+\alpha+1)}{\Gamma(\alpha+1) \Gamma(\lambda+1)} \mathrm{F}\left(-\lambda, \lambda+\alpha+\beta+1 ; \alpha+1 ; \frac{1-\mathrm{x}}{2}\right), \mathrm{x} \varepsilon(-1,1],
$$

where $\alpha, \beta>-1, \lambda \varepsilon \mathbf{R}$ and $\lambda+\alpha+1 \neq 0,-1,-2, \ldots$. We note that if $-1<\beta<1$, then $\mathrm{P}_{\lambda}^{(\alpha, \beta)}(\mathrm{x})$ is wel1-defined at $\mathrm{x}=-1$.

Since $\mathrm{P}_{\lambda}^{(\alpha, \beta)}(\mathbf{x})=\frac{\Gamma(\alpha-\lambda+1) \Gamma(\lambda-\alpha-\beta)}{\Gamma(1-\lambda) \Gamma(\lambda-\beta)} \mathbf{P}_{\lambda-\alpha-\beta-1}^{(\alpha, \beta)}(\mathbf{x})$, we may restrict ourselves, throughout the paper to the case $\lambda \geq-\frac{\alpha+\beta+1}{2}$. The function $\mathrm{P}_{\lambda}^{(\alpha, \beta)}(\mathrm{x})$ satisfies the differential equation

$$
\left(1-x^{2}\right) y^{\prime \prime}+(\beta-\alpha-(\alpha+\beta+2) x) y^{\prime}+\lambda(\lambda+\alpha+\beta+1) y=0
$$

We derive in the following lemmas basic relations that hold for the function $P_{\lambda}^{(\alpha, \beta)}(x)$ and are essential in the study of the transform. We note that most of these relations are generalizations of the case when $\lambda \varepsilon P$, where $P$ is the set of non-negative integers.

LEMMA 2.1. For any $x \in(-1,1]$ and any $\lambda \geq-\frac{\alpha+\beta+1}{2}$, the following relations hold

$$
\begin{aligned}
& \text { (i) }\left(1-x^{2}\right) \frac{d}{d x} P_{\lambda}^{(\alpha, \beta)}(x)=\left(\frac{\lambda(\alpha-\beta)}{2 \lambda+\alpha+\beta}-\lambda x\right) P_{\lambda}^{(\alpha, \beta)}(x)+\frac{2(\lambda+\alpha)(\lambda+\beta)}{2 \lambda+\alpha+\beta} P_{\lambda-1}^{(\alpha, \beta)}(x) ; \\
& \text { (ii) } \frac{d}{d x}\left((1-x)^{\alpha+1}(1+x)^{\beta+1} \frac{d}{d x} P_{\lambda}^{(\alpha, \beta)}(x)\right)=-\lambda(\lambda+\alpha+\beta+1)(1-x)^{\alpha}(1+x)^{\beta} P_{\lambda}^{(\alpha, \beta)}(x) ; \\
& \text { (iii) } P_{\lambda}^{(\alpha, \beta)}(1)=\frac{\Gamma(\lambda+\alpha+1)}{\Gamma(\alpha+1) \Gamma(\lambda+1)} ; \\
& \text { (iv) } \frac{d}{d x} P_{\lambda}^{(\alpha, \beta)}(1)=\frac{\lambda(\lambda+\alpha+\beta+1) \Gamma(\lambda+\alpha+1)}{2 \Gamma(\alpha+2) \Gamma(\lambda+1)} \text {. }
\end{aligned}
$$

PROOF. (i) Applying (H2), we may write $\mathrm{P}_{\lambda}^{(\alpha, \beta)}$ (x) as 


$$
\mathrm{P}_{\lambda}^{(\alpha, \beta)}(\mathrm{x})=\frac{\Gamma(\lambda+\alpha+1)}{\mathrm{I}^{\prime}(\alpha+1) \Gamma(\lambda+1)}\left(\frac{1+\mathrm{x}}{2}\right){ }_{\mathrm{F}}\left(-\lambda ;-\lambda-\beta ; \alpha+1 ; \frac{\mathrm{x}-1}{\mathrm{x}+1}\right) .
$$

Differentiating this expression, we obtain by (H7)

$$
\begin{gathered}
\frac{d}{d t} P_{\lambda}^{(\alpha, \beta)}(x)=\frac{\lambda}{2}\left(\frac{x+1}{2}\right)^{-1} P_{\lambda}^{(\alpha, \beta)}(x)+\frac{\Gamma(\lambda+\alpha+1)}{\Gamma(\alpha+1) \Gamma(\lambda+1)} \frac{\lambda(\lambda+\beta)}{2(\alpha+1)} \times \\
\left(\frac{x+1}{2}\right)^{\lambda-2} F\left(1-\lambda, 1-\lambda-\beta ; \alpha+2 ; \frac{x-1}{x+1}\right) .
\end{gathered}
$$

By means of (H4) and (H6), the last term can be written as a combination of $F\left(1-\lambda, 1-\lambda-\beta ; \alpha+1 ; \frac{x-1}{x+1}\right)$ and $F\left(-\lambda,-\lambda-\beta ; \alpha+1 ; \frac{x-1}{x+1}\right)$ which reduce to $P_{\lambda-1}^{(\alpha, \beta)}(x)$ and $\mathrm{P}_{\lambda}^{(\alpha, \beta)}(\mathrm{x})$ respectively.

Formula (ii) follows from differentiating the left-hand side and then using (2.3). The evaluations of (iii) and (iv) are immediate.

LEMMA 2.2. For any $x \in(-1,1]$ and $\lambda \geq-\frac{\alpha+\beta+1}{2}$, we have

(i) for $-1<\beta \leq 0,\left|P_{\lambda}^{(\alpha, \beta)}(x)\right| \leq \frac{\Gamma(\lambda+\alpha+1)}{\Gamma(\alpha+1) \Gamma(\lambda+1)}+M(\lambda, \alpha, \beta) \log \frac{2}{1+x}$

(ii) for $\beta \geq 0,\left(\frac{1+x}{2}\right)^{\beta}\left|P_{\lambda}^{(\alpha, \beta)}(x)\right| \leq \frac{\Gamma(\lambda+\alpha+1)}{\Gamma(\alpha+1) \Gamma(\lambda+1)}+M^{\prime}(\lambda, \alpha, \beta) \log \frac{2}{1+x}$

where $M(\lambda, \alpha, \beta)$ and $M^{\prime}(\lambda, \alpha, \beta)$ are constants depending upon $\alpha, \beta$ and $\lambda$.

PROOF. (i) We first observe that for $\lambda \geq-\frac{\alpha+\beta+1}{2}$, we have

$$
\frac{\Gamma(\lambda+\alpha+1)}{\Gamma(\alpha+1) \Gamma(\lambda+1)}\left|\frac{(-\lambda)_{k}(\lambda+\alpha+\beta+1)}{(\alpha+1)_{k} k !}\right| \leq M(\lambda, \alpha, \beta) k^{\beta-1}, k=1,2,3, \ldots
$$

for some constant $M(\lambda, \alpha, \beta)>0$. Since $-1<\beta \leq 0$, it follows that

$$
\left|P_{\lambda}^{(\alpha, \beta)}(x)\right| \leq \frac{\Gamma(\lambda+\alpha+1)}{\Gamma(\alpha+1) \Gamma(\lambda+1)}+M(\lambda, \alpha, \beta) \sum_{k=1}^{\infty} k^{-1}\left(\frac{1-x}{2}\right) k
$$

or

$$
\left|P_{\lambda}^{(\alpha, \beta)}(x)\right| \leq \frac{\Gamma(\lambda+\alpha+1)}{\Gamma(\alpha+1) \Gamma(\lambda+1)}+M(\lambda, \alpha, \beta) \log \frac{2}{1+x}
$$

(ii) From (H1) it follows that

$$
\left(\frac{1+x}{2}\right) \beta_{P_{\lambda}}^{(\alpha, \beta)}(x)=\frac{\Gamma(\lambda+\alpha+1)}{\Gamma(\alpha+1) \Gamma(\lambda+1)} F\left(\lambda+\alpha+1,-\lambda-\beta ; \alpha+1 ; \frac{1-x}{2}\right) .
$$

Again, observe that for $\lambda \geq-\frac{\alpha+\beta+1}{2}$, we have 


$$
\frac{\Gamma(\lambda+\alpha+1)}{\Gamma(\alpha+1) \Gamma(\lambda+1)}\left|\frac{(\lambda+\alpha+1) k^{(-\lambda-\beta)} k}{(\alpha+1) k^{k !}}\right| \leq M^{\prime}(\lambda, \alpha, \beta) k^{-(\beta+1)},
$$

for some constant $M^{\prime}(\lambda, \alpha, \beta)>0$. Since $\beta \geq 0$, it follows that

$$
\left(\frac{1+x}{2}\right)^{\beta}\left|P_{\lambda}^{(\alpha, \beta)}(x)\right| \leq \frac{\Gamma(\lambda+\alpha+1)}{\Gamma(\alpha+1) \Gamma(\lambda+1)}+M^{\prime}(\lambda, \alpha, \beta) \log \frac{2}{1+x} .
$$

An immediate consequence of Lemma 2.2 is that for any $\beta>-1$ we have

$$
\lim _{x \rightarrow-1}{ }^{(1+x)^{\beta+1}} \mathrm{P}_{\lambda}^{(\alpha, \beta)}(x)=0 .
$$

A relation of type $(2.4)$ is needed for the next lemma.

LEMMA 2.3. For any $\lambda \geq-\frac{\alpha+\beta+1}{2}$, we have

$$
\lim _{x \rightarrow-1}(1+x)^{\beta+1} \frac{d}{d x} P_{\lambda}^{(\alpha, \beta)}(x)=\frac{2^{\beta} \Gamma(\lambda+\alpha+1) \Gamma(\beta+1)}{\pi \Gamma(\lambda+\alpha+\beta+1)} \sin \pi \lambda .
$$

PROOF. Introduce first the function

$$
\mathbf{R}_{\lambda}^{(\alpha, \beta)}(\mathbf{x})=\frac{\Gamma(\alpha+1) \Gamma(\lambda+1)}{\Gamma(\lambda+\alpha+1)} \mathrm{P}_{\lambda}^{(\alpha, \beta)}(\mathrm{x})
$$

From lemma 2.1 (i), we have

$$
\begin{aligned}
\left(1-x^{2}\right) \frac{d}{d x} R_{\lambda}^{(\alpha, \beta)}(x)+\lambda(x+1) R_{\lambda}^{(\alpha, \beta)}(x)= & \frac{2 \lambda(\lambda+\alpha)}{2 \lambda+\alpha+\beta} R_{\lambda}^{(\alpha, \beta)}(x)+ \\
& \frac{2(\lambda+\alpha)(\lambda+\beta)}{2 \lambda+\alpha+\beta} R_{\lambda-1}^{(\alpha, \beta)}(x) .
\end{aligned}
$$

An application of (H2) and (H6) together with multiplication by $(1+x)^{\beta}$ yields

$$
(1-x)(1+x)^{\beta+1} \frac{d}{d x} R_{\lambda}^{(\alpha, \beta)}(x)+\lambda(x+1)^{\beta+1} R_{\lambda}^{(\alpha, \beta)}(x)=2^{\beta+1} \lambda F\left(\lambda+\alpha,-\lambda-\beta ; \alpha+1 ; \frac{1+x}{2}\right) .
$$

From (2.2) and (2.4) we obtain

$$
\lim _{x \rightarrow-1}(1+x)^{\beta+1} \frac{d}{d x} R_{\lambda}^{(\alpha, \beta)}(x)=\frac{2^{\beta} \Gamma(\alpha+1) \Gamma(\beta+1) \Gamma(\lambda+1) \sin \pi \lambda}{\pi \Gamma(\lambda+\alpha+\beta+1)} .
$$

Therefore,

$$
\lim _{x \rightarrow-1}(1+x)^{\beta+1} \frac{d}{d x} P_{\lambda}^{(\alpha, \beta)}(x)=\frac{2^{\beta} \Gamma(\lambda+\alpha+1) \Gamma(\beta+1) \sin \pi \lambda}{\pi \Gamma(\lambda+\alpha+\beta+1)} .
$$

Throughout the paper, we denote by $\mathrm{L}_{w}^{p}(-1,1), p \geq 1$, the space of all functions f for which $\|f\|_{p}$ given by 


$$
\left.\|f\|_{p}=\left(\frac{1}{2^{\alpha+\beta+1}}\right) \int_{-1}^{1}(1-x)^{\alpha}(1+x)^{\beta}|f(x)|^{p} d x\right)^{\frac{1}{p}}
$$

is finite. When the weight function $w(x) \equiv(1-x)^{\alpha}(1+x)^{\beta}$ is identically equal to 1 , we denote the space by $\mathrm{L}^{\mathrm{p}}(-1,1)$.

LEMMA 2.4. $\mathrm{P}_{\lambda}^{(\alpha, \beta)}(\mathrm{x}) \in \mathrm{L}_{\mathrm{w}}^{\mathrm{p}}(-1,1)$ for all $\mathrm{p} \geq 1$ and for all $\alpha$ and $\beta$ such that $\alpha p+1>0$ and $-\frac{1}{\mathrm{p}}<\beta<\frac{1}{\mathrm{p}}$.

PROOF. We first note that $\log \frac{2}{1+x} \varepsilon L^{q}(-1,1)$ for any $q \geq 1$ and hence $f(x)=\frac{\Gamma(\lambda+\alpha+1)}{\Gamma(\alpha+1) \Gamma(\lambda+1)}+M(\lambda, \alpha, \beta) \log \frac{2}{1+x}$ belongs to $L^{q}(-1,1)$ for any $q \geq 1$. Thus from Lemma 2.2 (i) we have by Hölder's inequality $\left(\frac{1}{p}+\frac{1}{q}=1, q=\frac{p}{p-1}\right)$,

$$
\begin{aligned}
\int_{-1}^{1}(1-x)^{\alpha}(1+x)^{\beta}\left|P_{\lambda}^{(\alpha, \beta)}(x)\right|^{p} d x & \leq\left(\int_{-1}^{1}(1-x)^{\alpha p}(1+x)^{\beta p} d x\right)^{\frac{1}{p}} \times \\
& \times\left(\int_{-1}^{1}\left|p_{\lambda}^{(\alpha, \beta)}(x)\right|^{p^{2} / p-1} d x\right)^{\frac{p-1}{p}} .
\end{aligned}
$$

Since $\frac{p^{2}}{p-1}>1$ for all $p>1$, it follows that the right-hand side is finite if $\alpha p+1>0$ and $\beta \mathrm{p}+1>0$. Thus $\mathrm{P}_{\lambda}^{(\alpha, \beta)}(\mathrm{x}) \varepsilon \mathrm{L}_{\mathrm{w}}^{\mathrm{p}}(-1,1)$, with $\alpha \mathrm{p}+1>0$ and $-\frac{1}{\mathrm{p}}<\beta \leq 0$.

From Lemma 2.2 (ii) with $\beta \geq 0$, we have

$$
\left|P_{\lambda}^{(\alpha, \beta)}(x)\right| \leq \frac{\Gamma(\lambda+\alpha+1)}{\Gamma(\alpha+1) \Gamma(\lambda+1)}\left(\frac{1+x}{2}\right)^{-\beta}+M^{\prime}(\lambda, \alpha, \beta)\left(\frac{1+x}{2}\right)^{-\beta} \log \frac{2}{1+x} .
$$

Using a similar argument as above we obtain

$$
\mathrm{P}_{\lambda}^{(\alpha, \beta)}(\mathrm{x}) \varepsilon \mathrm{L}_{\mathrm{w}}^{\mathrm{p}}(-1,1) \text { with } \alpha \mathrm{p}+1>0 \text { and } 0 \leq \beta<\frac{1}{\mathrm{p}} \text {. }
$$

The re fore, $\mathrm{P}_{\lambda}^{(\alpha, \beta)}(\mathrm{x}) \in \mathrm{L}_{\mathrm{w}}^{\mathrm{p}}(-1,1)$ with $\mathrm{p} \geq 1, \alpha \mathrm{p}+1>0,-\frac{1}{\mathrm{p}}<\beta<\frac{1}{\mathrm{p}}$.

Another useful lemma is:

LEMMA 2.5. Let $\lambda, v \geq-\frac{\alpha+\beta+1}{2}, \lambda \neq v \quad \lambda \neq-(v+\alpha+\beta+1)$ and $-\frac{1}{2}<\alpha<\frac{1}{2},-\frac{1}{2}<\beta<\frac{1}{2}$. Then

$$
\begin{aligned}
& \frac{1}{2^{\alpha+\beta+1}} \int_{-1}^{1}(1-x){ }^{\alpha}(1+x){ }^{\beta} P_{\lambda}^{(\alpha, \beta)}(x) P_{\nu}^{(\beta, \alpha)}(-x) d x \\
& \quad=\frac{\Gamma(\lambda+\alpha+1) \Gamma(\nu+\beta+1)}{\pi(\lambda-\nu)(\lambda+\nu+\alpha+\beta+1)}\left\{\frac{\sin \pi \lambda}{\Gamma(\nu+1) \Gamma(\lambda+\alpha+\beta+1)}-\frac{\sin \pi \nu}{\Gamma(\lambda+1) \Gamma(\nu+\alpha+\beta+1)}\right\} .
\end{aligned}
$$

PROOF. We first note that the integral is absolutely convergent since $\mathrm{P}_{\lambda}^{(\alpha, \beta)}(\mathrm{x})$ and $\mathrm{P}_{\lambda}^{(\beta, \alpha)}(-\mathrm{x}) \varepsilon \mathrm{L}_{\mathrm{w}}^{2}(-1,1)$ for $-\frac{1}{2}<\alpha<\frac{1}{2},-\frac{1}{2}<\beta<\frac{1}{2}$. Lemma 2.1 (ii), Lemma 2.4 
and an integration by parts yield

$$
\begin{aligned}
& -\lambda(\lambda+\alpha+\beta+1) \int_{-1}^{1}(1-x)^{\alpha}(1+x){ }^{\beta} P_{\lambda}^{(\alpha, \beta)}(x) P_{\nu}^{(\beta, \alpha)}(-x) d x \\
& =\left.(1-x)^{\alpha+1}(1+x)^{\beta+1} \frac{d}{d x} P_{\lambda}^{(\alpha, \beta)}(x) P_{v}^{(\beta, \alpha)}(-x)\right|_{-1^{+}} ^{1^{-}} \\
& -\int_{-1}^{1}(1-x)^{\alpha+1}(1+x)^{\beta+1} \frac{d}{d x} P_{\lambda}^{(\alpha, \beta)}(x) \frac{d}{d x} P_{\nu}^{(\beta, \alpha)}(-x) d x \\
& =-\frac{2^{\alpha+\beta+1} \Gamma(\lambda+\alpha+1) \Gamma(\nu+\beta+1) \sin \pi \lambda}{\pi \Gamma(\nu+1) \Gamma(\lambda+\alpha+\beta+1)}-\left.(1-x)^{\alpha+1}(1+x)^{\beta+1} P_{\lambda}^{(\alpha, \beta)}(x) \frac{d}{d x} P_{\nu}^{(\beta, \alpha)}(-x)\right|_{-1} ^{1^{-}}+ \\
& +\int_{-1}^{1} \mathrm{P}_{\lambda}^{(\alpha, \beta)}(\mathrm{x}) \frac{\mathrm{d}}{\mathrm{dx}}\left((1-\mathrm{x})^{\alpha+1}(1+\mathrm{x})^{\beta+1} \frac{\mathrm{d}}{\mathrm{dx}} \mathrm{P}_{\nu}^{(\beta, \alpha)}(-\mathrm{x})\right) \mathrm{dx} \\
& =-\frac{2^{\alpha+\beta+1} \Gamma(\lambda+\alpha+1) \Gamma(\nu+\beta+1) \sin \pi \lambda}{\pi \Gamma(\nu+1) \Gamma(\lambda+\alpha+\beta+1)}+\frac{2^{\alpha+\beta+1} \Gamma(\lambda+\alpha+1) \Gamma(\nu+\beta+1) \sin \pi \nu}{\pi \Gamma(\lambda+1) \Gamma(\nu+\alpha+\beta+1)} \\
& -v(\nu+\alpha+\beta+1) \int_{-1}^{1}(1-x)^{\alpha}(1+x){ }_{p}^{\beta}{ }_{\lambda}^{(\alpha, \beta)}(x) P_{\nu}^{(\beta, \alpha)}(-x) d x .
\end{aligned}
$$

Therefore

$$
\begin{aligned}
& \frac{1}{2^{\alpha+\beta+1}} \int_{-1}^{1}(1-x)^{\alpha}(1+x){ }^{\beta} P_{\lambda}^{(\alpha, \beta)}(x) P_{\nu}^{(\beta, \alpha)}(-x) d x \\
& =\frac{\Gamma(\lambda+\alpha+1) \Gamma(\nu+\beta+1)}{\pi(\lambda-\nu)(\lambda+\nu+\alpha+\beta+1)}\left\{\frac{\sin \pi \lambda}{\Gamma(\nu+1) \Gamma(\lambda+\alpha+\beta+1)}-\frac{\sin \pi \nu}{\Gamma(\lambda+1) \Gamma(\nu+\alpha+\beta+1)}\right\} .
\end{aligned}
$$

Before we proceed to obtain some estimates on $\mathrm{P}_{\lambda}^{(\alpha, \beta)}(\mathrm{x})$ for large $\lambda$, we collect some elementary properties of the Jacobi polynomials which are necessary in the development of the paper. For $\lambda=n \varepsilon P$, the series in (2.1) reduces to the classical Jacobi polynomials $\mathrm{P}_{\mathrm{n}}^{(\alpha, \beta)}(\mathbf{x})$ of degree $n$. There holds the orthogonality relation

$$
\frac{1}{2^{\alpha+\beta+1}} \int_{-1}^{1}(1-x)^{\alpha}(1+x){ }_{P_{n}}^{(\alpha, \beta)}(x) P_{m}^{(\alpha, \beta)}(x) d x= \begin{cases}0 & n \neq m \\ \delta_{n} & n=m\end{cases}
$$

where,

$$
\delta_{n}=\frac{\Gamma(n+\alpha+1) \Gamma(n+\beta+1)}{n !(2 n+\alpha+\beta+1) \Gamma(n+\alpha+\beta+1)}
$$


The discrete Jacobi transform of a function $f(x), x \varepsilon(-1,1)$ denoted by $\hat{\mathbf{f}}^{(\alpha, \beta)}(\mathbf{n})$ (see Debnath [3]) is

$$
\hat{\mathrm{f}}^{(\alpha, \beta)}(\mathrm{n})=\mathrm{J}(\mathrm{f}(\mathrm{x}))=\frac{1}{2^{\alpha+\beta+1}} \int_{-1}^{1}(1-x)^{\alpha}(1+x)^{\beta_{\mathrm{P}}} \mathrm{n}^{(\alpha, \beta)}(\mathrm{x}) \mathrm{f}(\mathrm{x}) \mathrm{dx},
$$

and $f(x)$ will then be given by

$$
f(x)=\sum_{n=0}^{\infty} \delta_{n}^{-1} \hat{f}^{(\alpha, \beta)}(n) P_{n}^{(\alpha, \beta)}(x)
$$

Moreover, if $\mathrm{f}(\mathrm{x}) \varepsilon \mathrm{L}_{\mathrm{w}}^{\mathrm{p}}(-1,1) \mathrm{p} \geq 1$, then $\hat{\mathrm{f}}^{(\alpha, \beta)}(\mathrm{n})$ defines a bounded linear mapping from $L_{W}^{p}(-1,1)$ into the space of all nu11 sequences. Thus one obtains the uniqueness theorem

$$
\hat{\mathbf{f}}^{(\alpha, \beta)}(\mathrm{n})=0 \Leftrightarrow \mathrm{f}(\mathrm{x})=0 \text { a.e. }
$$

We also note that for any $f, g_{2} L_{2}^{2}(-1,1)$, we have for the appropriate choice of $\alpha$ and $\beta$,

$$
\frac{1}{2^{\alpha+\beta+1}} \int_{-1}^{1}(1-x)^{\alpha}(1+x)^{\beta} f(x) g(x) d x=\sum_{n=0}^{\infty} \delta_{n}^{-1} \hat{f}^{(\alpha, \beta)}(n) \hat{g}^{(\alpha, \beta)}(n) .
$$

From (2.6) and Lemma 2.5 together with the identity $P_{n}^{(\alpha, \beta)}(-x)=(-1){ }_{n}^{n}(\beta, \alpha)(x)$, we obtain

$$
\hat{\mathrm{P}}_{\lambda}^{(\alpha, \beta)}(\mathrm{n})= \begin{cases}\frac{(-1)^{\mathrm{n}} \Gamma(\lambda+\alpha+1) \Gamma(\mathrm{n}+\beta+1) \sin \pi \lambda}{\pi(\lambda-\mathrm{n})(\lambda+\mathrm{n}+\alpha+\beta+1) n ! \Gamma(\lambda+\alpha+\beta+1)}, & \lambda \neq \mathrm{n} \\ \frac{\Gamma(\mathrm{n}+\alpha+1) \Gamma(\mathrm{n}+\beta+1)}{(2 \mathrm{n}+\alpha+\beta+1) n ! \Gamma(n+\alpha+\beta+1)}, & \lambda=\mathbf{n} .\end{cases}
$$

Since $P_{\lambda}^{(\alpha, \beta)}(x) \varepsilon L_{W}^{2}(-1,1),\left(\alpha>-\frac{1}{2},-\frac{1}{2}<\beta<s_{2}\right)$, it follows that

$$
\left\|P_{\lambda}^{(\alpha, \beta)}(x)\right\|_{2}^{2}= \begin{cases}\sum_{n=0}^{\infty} \delta_{n}^{-1}\left(\frac{\Gamma(\lambda+\alpha+1) \Gamma(n+\beta+1) \sin \pi \lambda}{\pi(\lambda-n)(\lambda+n+\alpha+\beta+1) n ! \Gamma(\lambda+\alpha+\beta+1)}\right)^{2}, & \lambda \neq n \\ \delta_{n} & , \lambda=n\end{cases}
$$

After this detour, we prove

LEMMA 2.6. For $\lambda, v \geq-\frac{\alpha+\beta+1}{2}, \alpha>-\frac{1}{2}$ and $-\frac{1}{2}<\beta<\frac{1}{2}$, we have

(i) for each compact subinterval $[a, b] \subset(-1,1)$, there holds for $x \varepsilon[a, b]$ and 


$$
\left|P_{\lambda}^{(\alpha, \beta)}(x)\right|=0\left(\lambda^{-\frac{1}{2}}\right)
$$

and

$$
\left\|\mathbf{P}_{\lambda}^{(\alpha, \beta)}(\mathbf{x})\right\|_{1} \leq\left\|\mathrm{P}_{\lambda}^{(\alpha, \beta)}(\mathbf{x})\right\|_{2}=0\left(\lambda^{-\frac{1}{2}}\right) ;
$$

(ii) for each $[c, d] \subset\left[-\frac{\alpha+\beta+1}{2}, \infty\right)$ there exists a constant $M>0$ such that for all $\lambda, v \varepsilon[c, d]$

$$
\left\|P_{\lambda}^{(\alpha, \beta)}(x)-P_{\nu}^{(\alpha, \beta)}(x)\right\|_{2} \leq M|\lambda-\nu|
$$

PROOF. (i) The estimate in (i) follows after some calculations from (formula 8, page 237, [7]). Actually for large $\lambda$, we obtain

$$
P_{\lambda}^{(\alpha, \beta)}(x)=\frac{2^{\alpha+\beta}}{(\lambda \pi)^{\frac{3}{2}}} \frac{\left(1+e^{-i \theta}\right)^{-\beta-\frac{1}{2}}}{\left(1-e^{-i \theta}\right)^{\alpha+\frac{1}{2}}}\left(e^{i \theta \lambda} \pm e^{ \pm i \pi\left(\alpha+\frac{1}{2}\right)-i \theta(\lambda+\alpha+\beta+1)}\right)\left(1+0\left(\left|\lambda^{-1}\right|\right)\right)
$$

where $\cos \theta=x$. From this we obtain the estimate.

(ii) We first consider the case when $\lambda, \nu \neq 0,1,2, \ldots$

$$
\begin{aligned}
& \left\|\mathrm{P}_{\lambda}^{(\alpha, \beta)}(\mathrm{x})-\mathrm{P}_{\nu}^{(\alpha, \beta)}(\mathrm{x})\right\|_{2^{2} \leq\left\|\mathrm{P}_{\lambda}^{(\alpha, \beta)}(x)\right\|_{2^{-}}^{2}} \\
& \quad-\frac{1}{2^{\alpha+\beta+1}} \int_{-1}^{1}(1-\mathrm{x})^{\alpha}(1+\mathrm{x}){ }^{\alpha} \mathrm{P}_{\lambda}^{(\alpha, \beta)}(\mathrm{x}) \mathrm{P}_{\nu}^{(\alpha, \beta)}(\mathrm{x}) \mathrm{d} \mathrm{x}+\left\|\mathrm{P}_{\nu}^{(\alpha, \beta)}(\mathrm{x})\right\|_{2}^{2}
\end{aligned}
$$

$(2.6),(2.7)$ and $(2.8)$ imply that

$$
\begin{gathered}
\left\|P_{\lambda}^{(\alpha, \beta)}(x)-P_{\nu}^{(\alpha, \beta)}(x)\right\|_{2}^{2} \leq \sum_{n=0}^{\infty} \delta_{n}^{-1}\left(\frac{\Gamma(n+\beta+1)}{m !}\right)^{2}\left(\frac{\Gamma(\lambda+\alpha+1) \sin \pi \lambda}{(\lambda-n)(\lambda+n+\alpha+\beta+1) \Gamma(\lambda+\alpha+\beta+1)}-\right. \\
\left.-\frac{\Gamma(\nu+\alpha+1) \sin \pi \nu}{(\nu-n)(\nu+n+\alpha+\beta+1) \Gamma(\nu+\alpha+\beta+1)}\right)^{2} .
\end{gathered}
$$

Set

$$
\phi(x)=\frac{\Gamma(x+\alpha+1)}{\Gamma(x+\alpha+\beta+1)}
$$

and

$$
\phi_{n}(x)=\phi^{\prime}(x) \frac{\sin \pi x}{(x-n)(x+n+\alpha+\beta+1)}+\phi(x) \frac{(x-n)(x+n+\alpha+\beta+1) \pi \cos \pi x-(2 x+\alpha+\beta+1) \sin \pi x}{(x-n)^{2}(x+n+\alpha+\beta+1)^{2}} .
$$

Clearly, $\phi(x)$ is continuously differentiable for $x>-\frac{\alpha+\beta+1}{2}$. Thus for any interval 
$[c, d] \subset\left(-\frac{\alpha+\beta+1}{2}, \infty\right), \phi_{n}(x)$ is continuous and remains bounded for any $x \neq n$. Moreover, by employing L' Hopital's rule we obtain that $\underset{x \rightarrow n}{\lim } \phi_{n}(x)$ exists and is equal to 0 . Now,

$$
\left\|P_{\lambda}^{(\alpha, \beta)}(x)-P_{\nu}^{(\alpha, \beta)}(x)\right\|_{2}^{2} \leq\left(\sum_{n=0}^{N}+\sum_{n=N+1}^{\infty}\right) \delta_{n}^{-1}\left(\frac{\Gamma(n+\beta+1)}{\pi n !}\right)^{2}\left(\int_{\nu}^{\lambda} \phi_{n}(x) d x\right)^{2}=S_{1}+S_{2},
$$

say, where $\mathrm{N}$ is chosen such that $\mathrm{N} \geq \max (|c|,|\mathrm{d}|)$.

$$
\left|s_{1}\right| \leq|\lambda-\nu|^{2} \sum_{n=0}^{N} \delta_{n}^{-1}\left(\frac{\Gamma(n+\beta+1)}{\pi n !}\right)^{2} \sup _{x \in[c, d]}\left|\phi_{n}(x)\right| \leq M_{N}|\lambda-\nu|^{2}
$$

for some $\mathrm{M}_{\mathrm{N}}>0$. Also,

$$
\begin{gathered}
\left|s_{2}\right| \leq|\lambda-\nu|^{2} \sum_{n=N+1}^{\infty} \delta_{n}^{-1}\left(\frac{\Gamma(n+\beta+1)}{m !}\right)^{2}\left\{\phi_{\max }^{\prime} \frac{1}{(n-N)(n-N+\alpha+\beta+1)}+\right. \\
\left.+\phi_{\max } \frac{(N+n)(N+\alpha+\beta+1) \pi+(2 N+\alpha+\beta+1)}{(n-N)^{2}(n-N+\alpha+\beta+1)^{2}}\right\}^{2}
\end{gathered}
$$

where, $\phi_{\max }=\max _{x \in[c, d]}|\phi(x)|$ and $\phi_{\max }^{\prime}=\max _{x \in[c, d]}\left|\phi^{\prime}(x)\right|$. Thus

$$
\left|\mathrm{S}_{2}\right| \leq \mathrm{M}_{N}^{*}|\lambda-\nu|^{2} \quad \text { for some } \mathrm{M}_{\mathrm{N}}^{*}>0 \text {. }
$$

Therefore, for $\lambda, v \varepsilon[c, d], \lambda, \nu \neq 0,1,2, \ldots$ and $M=\max \left(M_{N}, M_{N}^{*}\right)$ we have,

$$
\left\|P_{\lambda}^{(\alpha, \beta)}(x)-P_{\nu}^{(\alpha, \beta)}(x)\right\|_{2} \leq M|\lambda-\nu|
$$

If either $\lambda$ or $\nu$ or both assume the values $0,1,2, \ldots$, then a similar but simpler argument as above may be applied. This completes the proof of the lemma.

\section{THE CONTINUOUS JACOBI TRANSFORM AND ITS BASIC PROPERTIES}

In this section we define the continuous Jacobi transform and study some of its basic properties. The idea is to replace the Jacobi polynomial in the discrete Jacobi transform by the Jacobi function. Thus, we define the continuous Jacobi transform of $\mathrm{f} \varepsilon \mathrm{L}_{\mathrm{w}}^{2}(-1,1)$, with $\alpha>-\frac{1}{2},-\frac{1}{2}<\beta<\frac{1}{2}$, by

$$
\hat{\mathbf{f}}^{(\alpha, \beta)}(\lambda)=\frac{1}{2^{\alpha+\beta+1}} \int_{-1}^{1}(1-x)^{\alpha}(1+x)^{\beta} P_{\lambda}^{(\alpha, \beta)}(x) f(x) d x .
$$

We note that if $\alpha=\beta=0$, then $\hat{\mathrm{f}}^{(\alpha, \beta)}$ ( $\lambda$ ) reduces to the continuous Legendre transform of Butzer, Stens and Wehrens [5]. Further, if $\lambda=n \varepsilon P$, then $\hat{\mathbf{f}}^{(\alpha, \beta)}(\lambda)$ reduces to the 
discrete Jacobi transform of Debnath [3].

LEMMA 3.1. For any $f(x) \varepsilon L_{w}^{2}(-1,1)$, we have

(i) $\left|\hat{\mathrm{f}}^{(\alpha, \beta)}(\lambda)\right|=0\left(\lambda^{-\frac{1}{2}}\right)$;

(ii) for $\mathrm{p}>2, \hat{\mathbf{f}}^{(\alpha, \beta)}\left(\cdot-\frac{\alpha+\beta+1}{2}\right) \varepsilon \mathrm{C}_{0}\left(\mathbf{R}^{+}\right) \cap \mathrm{L}^{\mathrm{p}}\left(\mathbf{R}^{+}\right)$.

PROOF. (i) Lemma 2.6 (i) together with the Cauchy-Schwartz inequality yields

$$
\left|\hat{\mathrm{f}}^{(\alpha, \beta)}(\lambda)\right| \leq\left\|\mathrm{P}_{\lambda}^{(\alpha, \beta)}(\mathrm{x})\right\|_{2}\|\mathrm{f}\|_{2}=0\left(\lambda^{-\frac{1}{2}}\right)
$$

(ii) Again, Lemma 2.6 (ii) together with the Cauchy-Schwartz inequality yields

$$
\left|\hat{\mathbf{f}}^{(\alpha, \beta)}(\lambda)-\hat{\mathrm{f}}^{(\alpha, \beta)}(\nu)\right| \leq \mathrm{M}|\lambda-\nu|\|\mathrm{f}\|_{2}
$$

Hence $\lim _{\lambda \rightarrow \nu}\left(\hat{\mathrm{f}}^{(\alpha, \beta)}(\lambda)-\hat{\mathrm{f}}^{(\alpha, \beta)}(\nu)\right)=0$. Therefore, $\hat{\mathrm{f}}^{(\alpha, \beta)}(\lambda)$ is continuous on $\left(-\frac{\alpha+\beta+1}{2}, \infty\right)$ or $\hat{\mathrm{f}}^{(\alpha, \beta)}\left(\cdot-\frac{\alpha+\beta+1}{2}\right) \varepsilon C_{0}\left(\mathbb{R}^{+}\right)$. Now, in order to prove that $\hat{\mathrm{f}}^{(\alpha, \beta)}\left(\cdot-\frac{\alpha+\beta+1}{2}\right) \varepsilon L^{\mathrm{p}}\left(\mathbf{R}^{+}\right), \mathrm{p}>2$, we need to show that

$$
\left\|\hat{\mathrm{f}}^{(\alpha, \beta)}\left(\cdot-\frac{\alpha+\beta+1}{2}\right)\right\|_{\mathrm{p}}^{\mathrm{p}}=\int_{0}^{\infty}\left|\hat{\mathrm{f}}^{(\alpha, \beta)}\left(\cdot-\frac{\alpha+\beta+1}{2}\right)\right|^{\mathrm{p}} \mathrm{d} \lambda<\infty
$$

For any fixed $\mathrm{T}>0$, we have, by (i) above and Lemma 2.6 (i),

$$
\begin{aligned}
& \left\|\hat{\mathrm{f}}^{(\alpha, \beta)}\left(\lambda-\frac{\alpha+\beta+1}{2}\right)\right\|_{\mathrm{p}}^{\mathrm{p}} \leq\|\mathrm{f}\|_{2}\left(\int_{0}^{\mathrm{T}}\left\|\mathrm{p}_{\lambda-\frac{\alpha+\beta+1}{2}}^{(\alpha, \beta)}(\mathrm{x})\right\|_{2}^{\mathrm{p}} \mathrm{d} \lambda+\int_{\mathrm{T}}^{\infty}\left\|\mathrm{P}_{\lambda-\frac{\alpha+\beta+1}{2}}^{(\alpha, \beta)}(\mathrm{x})\right\|_{2}^{\mathrm{p}} \mathrm{d} \lambda\right) \\
& \leq\|\mathrm{f}\|_{2}\left(\mathrm{C}_{1}+\mathrm{c}_{2} \int_{\mathrm{T}}^{\infty}\left|\lambda-\frac{\alpha+\beta+1}{2}\right|^{-\mathrm{p} / 2} \mathrm{~d} \lambda\right)^{1 / \mathrm{p}}
\end{aligned}
$$

where $C_{1}$ and $C_{2}$ are some positive constants. It follows that $\left\|\hat{f}(\alpha, \beta)\left(\lambda-\frac{\alpha+\beta+1}{2}\right)\right\|_{p}^{p}<\infty$ if $\mathrm{p}<2$. Thus $\hat{\mathrm{f}}^{(\alpha, \beta)}\left(\cdot-\frac{\alpha+\beta+1}{2}\right) \varepsilon \mathrm{C}_{\mathrm{p}}\left(\mathbf{R}^{+}\right) \cap \mathrm{L}^{\mathrm{p}}\left(\mathbf{R}^{+}\right)$.

LEMMA 3.2. Let $F(x)$ be a function defined on $[0, \infty)$ such that $\lambda^{\alpha+\beta+\frac{1}{2}} F(\lambda) \varepsilon L^{1}\left(\mathbf{R}^{+}\right)$. Then

$$
G(x)=\int_{0}^{\infty} F(\lambda) P P^{(\beta, \alpha)} \frac{\alpha+\beta+1}{2}(-x) H(\lambda) \lambda \sin \pi \lambda d \lambda
$$

belongs to $C(-1,1) \cap L_{w}^{2}(-1,1)$, where $H(\lambda)$ is given by

$$
H(\lambda)=\frac{\Gamma^{2}\left(\lambda+\frac{\alpha+\beta+1}{2}\right)}{\Gamma\left(\lambda+\frac{\alpha-\beta+1}{2}\right) \Gamma\left(\lambda+\frac{\beta-\alpha+1}{2}\right)} .
$$


PROOF. Observe that $H(\lambda)$, for large $\lambda$, behaves like $\lambda^{\alpha+\beta}\left(1+0\left(\lambda^{-1}\right)\right)$ (see [7]). For any fixed $T>0$, we have by Lemma 2.6 (ii) that

$$
\begin{aligned}
|G(x)| \leq\left|\int_{0}^{T} F(\lambda) P \underset{\lambda-\frac{\alpha+\beta+1}{2}}{(-x) H(\lambda) \lambda \sin \pi \lambda d \lambda|+| \int_{T}^{\infty} F(\lambda) P} \lambda-\frac{\alpha+\beta+1}{2}(-x) H(\lambda) \lambda \sin \pi \lambda d \lambda\right| \\
\quad \leq A_{1}+A_{2} \int_{T}^{\infty} \lambda^{\alpha+\beta+\frac{1}{2}}|F(\lambda)| d \lambda
\end{aligned}
$$

where $A_{1}$ and $A_{2}$ are some positive constants. Thus by the hypothesis, $G(x)$ is welldefined.

We first show that $G(x) \varepsilon C(-1,1)$. For any $x \in(-1,1)$, there exists a sufficiently small $\delta_{1}$ such that $x \pm \delta_{1} \varepsilon(-1,1)$. By Lemma 2.6 (i) and for all $|y|<\delta_{1}$, we have

$$
\begin{aligned}
|G(x+y)-G(x)| & \leq \int_{0}^{\infty} \lambda|F(\lambda)| H(\lambda)\left|P_{\lambda-\frac{\alpha+\beta+1}{2}}^{(\beta, \alpha)}(-x-y)-\frac{P}{\lambda-\frac{\alpha+\beta+1}{2}}(-x)\right| d \lambda \\
& \leq 2 M \int_{0}^{\infty} \lambda^{\alpha+\beta+\frac{1}{2}}|F(\lambda)| d \lambda<\infty
\end{aligned}
$$

by hypothesis and $M$ is some positive constant. Thus for $\lambda_{0}$ sufficientlv large

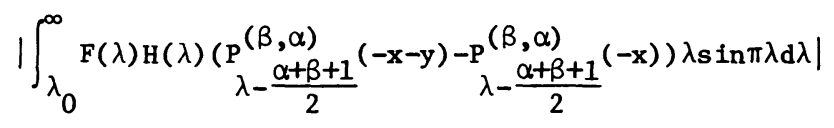

can be made sufficiently small. That is, given $\varepsilon>0$, there exists a $\lambda_{0}$ sufficientlv large such that the integral in (3.1) is less than $\varepsilon / 2$. Fix $\lambda_{0}$. By the continuity of $\mathrm{P}_{\lambda-\frac{\alpha+\beta+1}{2}}^{(\beta, \alpha)}$ over $(-1,1)$, we have for $\hat{\varepsilon}>0$ that there exists a $\delta_{2}>0$ such that

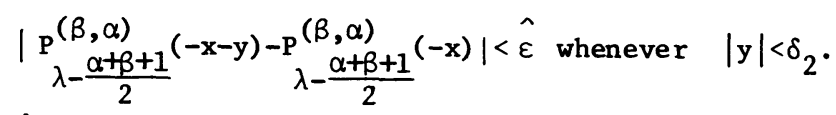

Choose $\frac{\varepsilon}{2}=\hat{\varepsilon} \int_{0}^{\lambda_{0}} \lambda^{\alpha+\beta+\frac{1}{2}}|F(\lambda)| d \lambda$ and $\delta=\min \left(\delta_{1}, \delta_{2}\right)$. Then $|G(x+y)-G(x)|<\varepsilon$ whenever $|y|<\delta$. Thus $G(x) \varepsilon C(-1,1)$.

We next show that $G(x) \varepsilon L_{w}^{2}(-1,1)$.

$$
\|G\|_{2}^{2}=\frac{1}{2^{\alpha+\beta+1}} \int_{-1}^{1}(1-x)^{\alpha}(1+x)^{\beta}\left|\int_{0}^{\infty} F(\lambda) P_{\lambda-\frac{\alpha+\beta+1}{2}}^{(\beta, \alpha)}(-x) \lambda(\lambda) \lambda \sin \pi \lambda d \lambda\right|^{2} d x
$$

and by Hardy-Littlewood-Polya inequality (see [8], page 148) and Lemma 2.6 (1), we obtain 


$$
\begin{aligned}
\|G\|_{2} & \leq \int_{0}^{\infty} \lambda|\mathrm{F}(\lambda)| \mathrm{H}(\lambda) \mathrm{d} \lambda\left(\frac{1}{2^{\alpha+\beta+1}} \int_{-\ddot{i}}^{1}(1-x)^{\alpha}(1+x)^{\beta}\left|\mathrm{P}_{\lambda-\frac{\alpha+\beta+1}{2}}^{(\beta, \alpha)}(-x)\right|^{2} \mathrm{dx}\right)^{\frac{1}{2}} \\
& \leq \mathrm{C} \int_{0}^{\infty} \lambda^{\alpha+\beta+\frac{1}{2}}|\mathrm{~F}(\lambda)| \mathrm{d} \lambda<\infty
\end{aligned}
$$

by hypothesis for some constant $C>0$. Thus $G(x) \varepsilon C(-1,1) \cap L_{w}^{2}(-1,1)$ and this completes the proof.

It can be easily deduced from Lemma 3.2 that

COROLLARY 3.1. If $\alpha+\beta=0 \quad\left(-\frac{1}{2}<\alpha<\frac{1}{2},-\frac{1}{2}<\beta<\frac{1}{2}\right)$ and $F(x)$ is such that $\lambda^{\frac{1}{2}} F(\lambda) \in L_{1}\left(\mathbb{R}^{+}\right)$, then

$$
G(x)=\int_{0}^{\infty} F(\lambda) P_{\lambda-\frac{1}{2}}^{(\beta, \alpha)}(-x) H_{0}(\lambda) \lambda \sin \pi \lambda d \lambda
$$

belongs to $C(-1,1) \cap L_{w}^{2}(-1,1)$ where

$$
\mathrm{H}_{0}(\lambda)=\frac{\Gamma^{2}\left(\lambda+\frac{1}{2}\right)}{\Gamma\left(\lambda+\alpha+\frac{1}{2}\right) \Gamma\left(\lambda+\beta+\frac{1}{2}\right)} .
$$

PROPOSITION 3.1. For $x \varepsilon(-1,1], \alpha+\beta=0,-\frac{1}{2}<\alpha, \beta<\frac{1}{2}$ and $k \varepsilon P$, we have

$$
\mathrm{P}_{\mathrm{k}}^{(\alpha, \beta)}(\mathrm{x})=4 \int_{0}^{\infty} \hat{\mathrm{P}}_{\mathrm{k}}^{(\alpha, \beta)}\left(\lambda-\frac{1}{2}\right) \mathrm{P}_{\lambda-\frac{1}{2}}^{(\beta, \alpha)}(-\mathrm{x}) \mathrm{H}_{0}(\lambda) \lambda \sin \pi \lambda \mathrm{d} \lambda .
$$

PROOF. For $\lambda>-\frac{1}{2}, \lambda \neq k+\frac{1}{2}$, we have from (2.7) with $\alpha+\beta=0$,

$$
\hat{\mathbf{P}}_{k}^{(\alpha, \beta)}\left(\lambda-\frac{1}{2}\right)=\hat{\mathbf{P}}_{\lambda-\frac{1}{2}}^{(\alpha, \beta)}(k)=\frac{(-1)^{k} \Gamma\left(\lambda+\alpha+\frac{1}{2}\right) \Gamma(k+\beta+1) \sin \pi\left(\lambda-\frac{1}{2}\right)}{\pi\left(\lambda-k-\frac{1}{2}\right)\left(\lambda+k+\frac{1}{2}\right) k ! \Gamma\left(\lambda+\frac{1}{2}\right)},
$$

so that $\lambda^{\frac{1}{2}} \hat{\mathrm{P}}_{\mathbf{k}}^{(\alpha, \beta)}\left(\lambda-\dot{x}_{2}\right) \varepsilon \mathrm{L}^{1}\left(\mathbf{R}^{+}\right)$. Denote the integral in (3.2) by $G_{k}(\mathbf{x})$. Then by Corollary 3.1, we have $G_{k}(x) \in C(-1,1) \cap L_{w}^{2}(-1,1)$. The idea now is to evaluate the discrete Jacobi transform of $G_{k}(x)$ and show that it is equal to $\hat{P}_{k}^{(\alpha, \beta)}(j)$ whence by (2.5) we obtain $G_{k}(x)=P_{k}^{(\alpha, \beta)}(x)$.

$$
\begin{aligned}
\hat{G}_{k}^{(\alpha, \beta)}(j) & =\frac{1}{2} \int_{-1}^{1}(1-x)^{\alpha}(1+x){ }^{\beta} P_{j}^{(\alpha, \beta)}(x) G_{k}(x) d x \\
& =2 \int_{-1}^{1}(1-x){ }^{\alpha}(1+x){ }_{P} P_{j}^{(\alpha, \beta)}(x) \int_{j}^{\infty} \hat{P}_{k}^{(\alpha, \beta)}\left(\lambda-\frac{1}{2}\right) P_{\lambda-\frac{1}{2}}^{(\beta, \alpha)}(-x) H_{0}(\lambda) \lambda \sin \pi \lambda d \lambda d x
\end{aligned}
$$

which by an application of Fubini's theorem, (3.3) and Lemma 2.5 yields 


$$
\hat{G}_{k}^{(\alpha, \beta)}(j)=\frac{(-1)^{k} 4 \Gamma(k+\beta+1) \Gamma(j+\alpha+1)}{\pi^{2} k ! j !} \int_{0}^{\infty} \frac{\lambda \sin \pi \lambda \sin ^{2} \pi\left(\lambda-\frac{1}{2}\right)}{\left(\lambda^{2}-\left(k+\frac{1}{2}\right)^{2}\right)\left(\lambda^{2}-\left(j+\frac{1}{2}\right)^{2}\right)} d \lambda .
$$

At this stage we can employ the method used in [5] to evaluate the above integral. In particular, we obtain that

$$
\hat{G}_{k}^{(\alpha, \beta)}(j)=\frac{(-1)^{k} \Gamma(k+\beta+1) \Gamma(j+\alpha+1)}{4 \pi^{2} k ! j !(k+j+1) i} \lim _{R \rightarrow \infty} \int_{-R}^{R}\left(g_{1}(\lambda)-g_{2}(\lambda)\right) d \lambda
$$

where

$$
\begin{aligned}
& g_{i}(z)=\frac{e^{i \pi z}+e^{i 3 \pi z}}{\left(z-k-\frac{1}{2}\right)\left(z-j-\frac{1}{2}\right)} \\
& g_{2}(z)=\frac{e^{-i \pi z}+e^{-i 3 \pi z}}{\left(z-k-\frac{1}{2}\right)\left(z-j-\frac{1}{2}\right)} .
\end{aligned}
$$

The method of [5] yields

$$
\hat{G}_{k}^{(\alpha, \beta)}(j)=\begin{array}{cc}
0 & j \neq k \\
\frac{\Gamma(k+\alpha+1) \Gamma(k+\beta+1)}{(k !)^{2}(2 k+1)}, & j=k
\end{array}
$$

Hence $\hat{G}_{k}^{(\alpha, \beta)}(j)=\hat{P}_{k}^{(\alpha, \beta)}(j)$. Therefore $G_{k}(x)=P_{i}^{(\alpha, \beta)}(x)$ by (2.5).

We are now ready to prove an inversion formula for the continuous Jacobi transform $\hat{f}^{(\alpha, \beta)}(x)$ of a function $f(x) \varepsilon L_{w}^{2}(-1,1)$. We are still assuming that $\alpha+\beta=0$.

THEOREM 3.1. Let $\mathrm{fEL}_{\mathrm{w}}^{2}(-1,1)$ be such that $\lambda^{\frac{1}{2}} \hat{\mathrm{f}}^{(\alpha, \beta)}\left(\lambda-\frac{1}{2}\right) \varepsilon \mathrm{L}^{1}\left(\mathbf{R}^{+}\right)$. Then for almost every $x \varepsilon(-1,1)$,

$$
f(x)=4 \int_{0}^{\infty} \hat{f}^{(\alpha, \beta)}\left(\lambda-\frac{1}{2}\right) P{ }_{\lambda-\frac{1}{2}}^{(\beta, \alpha)}(-x) H_{0}(\lambda) \lambda \sin \pi \lambda d \lambda .
$$

In addition, if $f$ is continuous on $(-1,1)$, then $(3.4)$ holds everywhere on $(-1,1)$.

PROOF. Denote the right-hand side of (3.4) by $J(x)$. Then by Corollary 3.1, $J(x) \in C(-1,1) \cap L_{w}^{2}(-1,1)$. The discrete Jacobi transform of $J(x)$ is

$$
\hat{J}^{(\alpha, \beta)}(x)=\frac{1}{2} \int_{-1}^{1}(1-x)^{\alpha}(1+x){ }^{\beta} P_{k}^{(\alpha, \beta)}(x) J(x) d x
$$




$$
=2 \int_{-1}^{1}(1-x)^{\alpha}(1+x)^{\beta} P_{k}^{(\alpha, \beta)}(x) \int_{0}^{\infty} \hat{f}^{(\alpha, \beta)}\left(\lambda-\frac{1}{2}\right) P_{\lambda-\frac{1}{2}}^{(\beta, \alpha)}(-x) H_{0}(\lambda) \lambda \sin \pi \lambda d \lambda d x .
$$

The definition of $\hat{\mathrm{f}}^{(\alpha, \beta)}\left(\lambda-\frac{1}{2}\right)$ and an application of Fubini's theorem yield

$$
\begin{aligned}
\hat{J}^{(\alpha, \beta)}(\mathrm{k}) & =4 \int_{0}^{\infty}\left(\frac{1}{2} \int_{-1}^{1}(1-y)^{\alpha}(1+y){ }_{P_{\lambda-\frac{1}{2}}}(\alpha, \beta)(y) f(y) d y\right) \times \\
& \times\left(\frac{1}{2} \int_{-1}^{1}(1-x)^{\alpha}(1+x){ }^{\beta} P_{k}^{(\alpha, \beta)}(x) P_{\lambda-\frac{1}{2}}^{(\beta, \alpha)}(-x) d x\right) H_{0}(\lambda) \lambda \sin \pi \lambda d \lambda \\
& =4 \int_{0}^{\infty} \lambda H_{0}(\lambda) \sin \pi \lambda\left(\frac{1}{2} \int_{-1}^{1}(1-y){ }^{\alpha}(1+y){ }_{P_{\lambda-\frac{1}{2}}}^{(\alpha, \beta)}(y) f(y) d y\right) \times \\
& \times\left[P_{k}^{(\alpha, \beta)}(\cdot)\right]_{(\beta, \alpha)}^{\Lambda}\left(\lambda-\frac{1}{2}\right) d \lambda
\end{aligned}
$$

where, in general,

$$
\begin{aligned}
& {[Q(-\cdot)]_{(\beta, \alpha)}^{\Lambda}(\lambda) \equiv \frac{1}{2} \int_{-1}^{1}(1-x){ }^{\beta}(1+x){ }^{\alpha} P_{\lambda}^{(\beta, \alpha)}(x) Q(-x) d x} \\
& =\frac{1}{2} \int_{-1}^{1}(1-x)^{\alpha}(1+x){ }^{\beta}{ }_{\lambda}^{(\beta, \alpha)}(-x) Q(x) d x .
\end{aligned}
$$

Now another application of Fubini's theorem together with Proposition 3.1 yields

$$
\begin{aligned}
& \hat{J}^{(\alpha, \beta)}(k)=\frac{1}{2} \int_{-1}^{1}(1-y)^{\alpha}(1+y)^{\beta} f(y)(-1)^{k}\left[4 \int_{0}^{\infty} \hat{p}_{k}^{(\beta, \alpha)}\left(\lambda-\frac{1}{2}\right) P_{\lambda-\frac{1}{2}}^{(\alpha, \beta)}(y) H_{0}(\lambda) \lambda \sin \pi \lambda d \lambda\right] d y \\
& =\frac{1}{2} \int_{-1}^{1}(1-y)^{\alpha}(1+y)^{\beta} f(y)(-1)^{k} P_{k}^{(\beta, \alpha)}(-y) d y \\
& =\frac{1}{2} \int_{-1}^{1}(1-y)^{\alpha}(1+y)^{\beta} P_{k}^{(\alpha, \beta)}(y) f(y) d y=\hat{f}^{(\alpha, \beta)}(k) \text {. }
\end{aligned}
$$

Now (2.5) implies that $f(x)=J(x)$ a.e., $x \in(-1,1)$. If, in addition, $f(x)$ is assumed to be continuous, then both sides of (3.4) are continuous and the above result will be valid everywhere in $(-1,1)$.

We end this section with a few remarks. We first note that if the inverse Jacobi transform given in (3.4) is denoted by $\underset{f}{v}(\alpha, \beta)$, then the inversion theorem 3.1 states that $f=\left[\hat{f}^{(\alpha, \beta)}\right]^{v(\alpha, \beta)}$. Under suitable conditions, it can be shown that $f=\left[v_{f}^{v}(\alpha, \beta)\right]^{\wedge}(\alpha, \beta)$. Furthermore, the restriction on $\alpha$ and $\beta$, namely, $-\frac{1}{2}<\alpha, \beta<\frac{1}{2}$ and $\alpha+\beta=0$ is necessary to effect the inversion formula. The special case $\alpha=\beta=0$ 
corresponds to the continuous Legendre transform developed by Butzer et. al [5].

The case of $\alpha=\beta=-\frac{m}{2}$ where $m$ is a non-negative integer requires a separate analysis and should lead to the continuous version of the associated Legendre transform [9].

We also note that the continuous Jacobi transform may be extended to distributions along the lines of Zemanian [10]. This will require the construction of a Fréchet space that contains the kernel $(1-x){ }^{\alpha}(1+x){ }^{\beta} P_{\lambda}(\alpha, \beta)(x)$ as an element. The transform will then be defined on the dual space as the application of the distribution to this kernel. This will be the subject of a later paper. ACKNOWLEDGEMENTS. The work of the second author was partially supported by NSERC of Canada under Grant A-7184 while he was on leave at the University of Petroleum and Minerals in Saudi Arabia. The authors would like to thank a referee for bringing reference [4] to their attention.

\section{REFERENCES}

[1] SNEDDON, I.N. The Use of Integral Transforms, McGraw Hil1, 1972 .

[2] JOHNSON, D.E. and JOHNSON, J.R., Mathematical Methods in Engineering Physics, Prentice-Hall, 1982.

[3] DEBnath, L. On Jacobi Transform, Bul1. Ca1. Math. Soc., Vol. 55 (1963), 113-120.

[4] DEBNATH, L. Solutions of Partial Differential Equations by the Method of Jacobi Transform, Bu11. Cal. Math. Soc. 59 (1967) 155-158.

[5] BUTZER, P.L., STENS, R.L. and WEHRENS, M., The Continuous Legendre Trans form, its Inverse Transform, and App1ications, Internat. J. Math. and Math. Sci., Vol. 3 , No. 1 (1980), 47-67.

[6] ERDELYI, A., MAGNUS, W., OBERHETTINGER, F and TRICOMI, F.G., Higher Transcendental Functions, Vo1. 1, McGraw-Hill Book Company, New York, 1953.

[7] LUKE, Y.L. The Special Functions and Their Applications, Academic Press, New York, 1969 .

[8] HARDY, G.H., LITTLEWOOD, J.E. and POLYA, G. Inequalities, Cambridge Univ. Press, Cambridge, 1964.

[9] DEBNATH, L., and C.W. HARRELI, The Operational Calculus of Associated Legendre Transform-I, Ind. J. Pure and App. Math., Vol. 7 (1976), 278-291.

[10] ZEMANIAN, A.H. Generalized Integral Transformations, John Wiley and Sons, New York, 1968. 


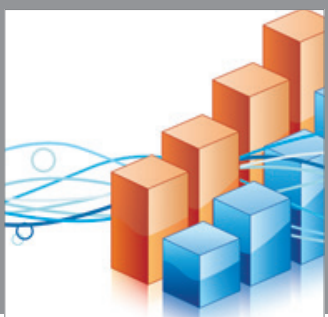

Advances in

Operations Research

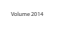

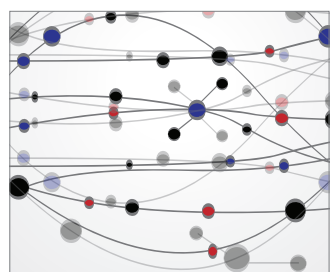

\section{The Scientific} World Journal
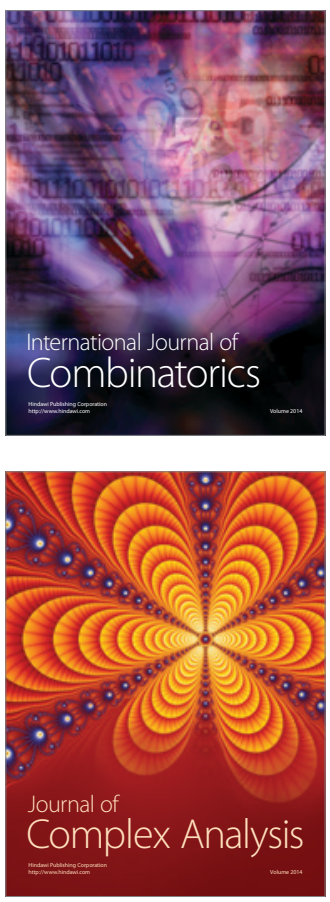

International Journal of

Mathematics and

Mathematical

Sciences
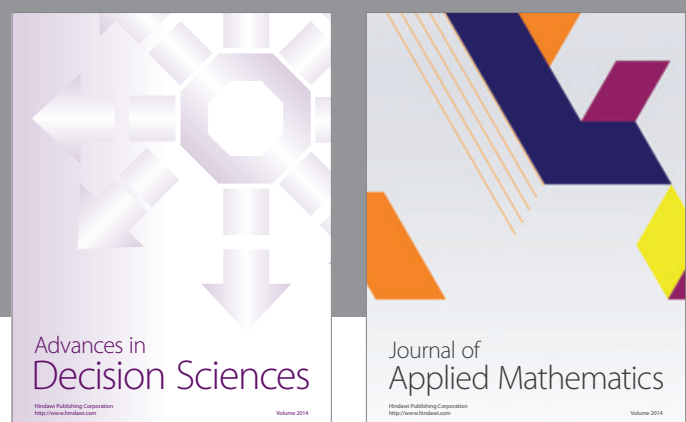

Journal of

Applied Mathematics
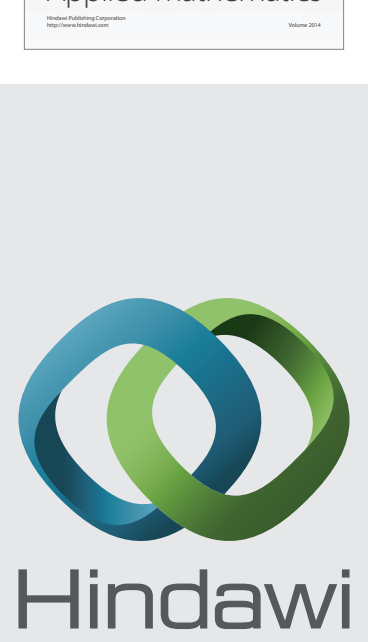

Submit your manuscripts at http://www.hindawi.com
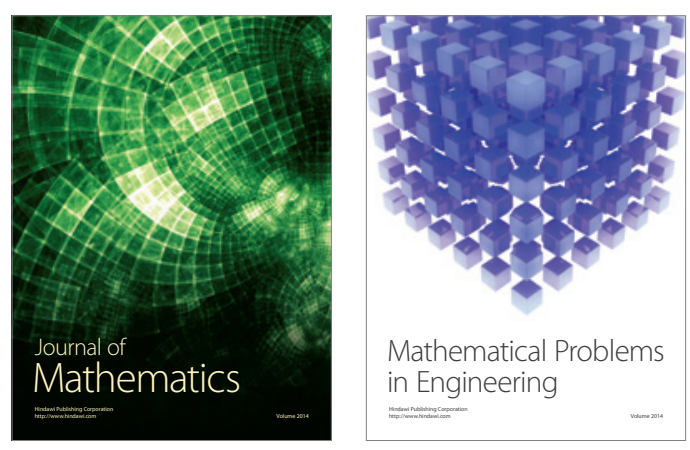

Mathematical Problems in Engineering
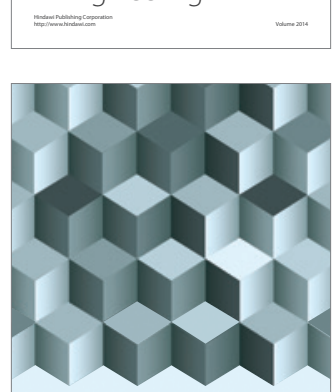

Journal of

Function Spaces
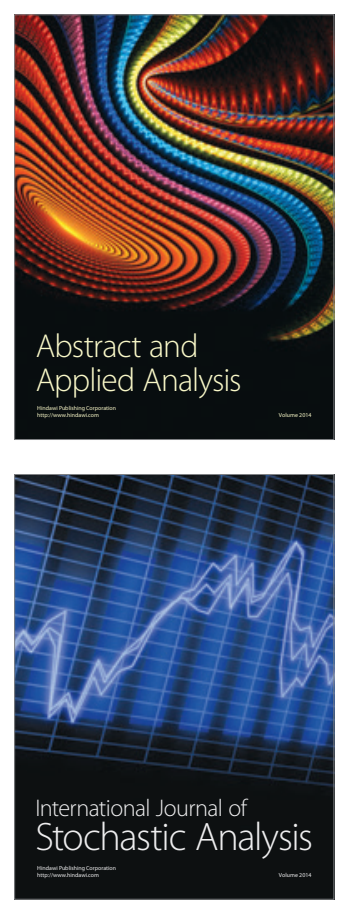

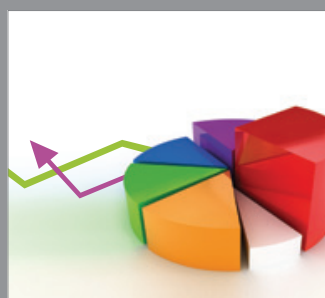

ournal of

Probability and Statistics

Promensencen
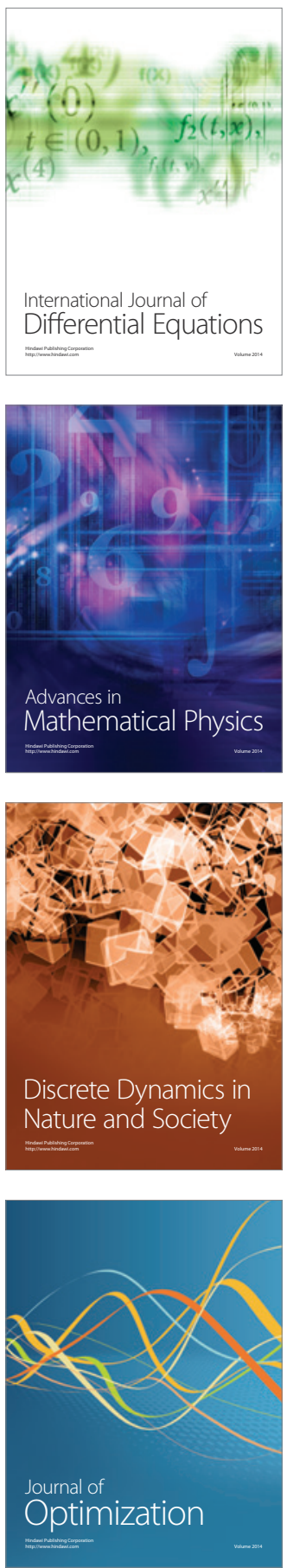